\title{
Optical absorption in lithiated tungsten oxide thin films: Experiment and theory
}

\author{
Lars Berggren, Jacob C. Jonsson, and Gunnar A. Niklasson ${ }^{a}$ \\ Department of Engineering Sciences, The Ångström Laboratory, Uppsala University, P.O. Box 534, \\ SE-751 21 Uppsala, Sweden
}

(Received 16 June 2007; accepted 30 August 2007; published online 25 October 2007)

\begin{abstract}
Amorphous tungsten oxide exhibits electrochromism when intercalated with protons, lithium, sodium, and other ions. Thin films of the material were prepared by dc magnetron sputtering and then electrochemically intercalated with lithium. The optical absorption in the wavelength range of $300-2500 \mathrm{~nm}$ was measured for a number of lithium concentrations. The optical absorption shows a maximum for lithium/tungsten ratios of 0.3-0.5. The optical spectra can be fitted by a superposition of three Gaussian peaks, representing the three possible electronic transitions between $\mathrm{W}^{6+}, \mathrm{W}^{5+}$, and $\mathrm{W}^{4+}$ sites. The variation of the peak strength with lithium concentration is consistent with an extended site-saturation theory. (C) 2007 American Institute of Physics.
\end{abstract}

[DOI: $10.1063 / 1.2800838]$

\section{INTRODUCTION}

Electrochromic (EC) devices are of great technological interest for a number of applications, ${ }^{1}$ for example, in energy-efficient "smart windows" applications. ${ }^{3}$ Thin films of EC materials are usually deposited onto glass or polymeric substrates that have been coated by a transparent conductive film. Optically efficient EC devices encompass a substrate with an anodic EC film and another one with a cathodic EC film, which are then laminated together by a polymer electrolyte. ${ }^{2}$

Amorphous tungsten oxide films have been found to exhibit excellent EC properties such as high coloration efficiency, good cyclic reversibility, and long lifetime. ${ }^{4,5}$ Deb first made a thorough study of electrochromism in amorphous or disordered tungsten trioxide. ${ }^{6}$ Until today, no other cathodic EC material has been able to compete with its favorable EC optical switching and good stability. The most convenient way to color a tungsten oxide film is by dual injection of electrons from the back contact and small positively charged ions, for example, protons, lithium, or sodium ones, from an electrolyte.

A detailed understanding of the optical processes responsible for the electrochromic effect would greatly facilitate the optimization of EC devices. It is well-known that the coloration (i.e., the optical absorption) of tungsten oxide is due to injected electrons that become positioned at W sites. Hence, these electrons alter the total valence of the tungsten ion from $\mathrm{W}^{6+}$ to $\mathrm{W}^{5+}$. Consequently, the optical absorption process has been modeled as an intervalence charge transfer of electrons from $\mathrm{W}^{5+}$ to $\mathrm{W}^{6+}$ sites. ${ }^{7}$ In stoichiometric amorphous tungsten oxide $\left(a-\mathrm{WO}_{3}\right)$, only $\mathrm{W}^{6+}$ ions are present, while it was assumed that substoichiometric as well as intercalated films also exhibit $\mathrm{W}^{5+}$ states. More recently, it has been argued that substoichiometric $a$ - $\mathrm{WO}_{y}(y<3)$ contains $\mathrm{W}^{4+}$ ions together with the $\mathrm{W}^{6+}$ ones. ${ }^{8-10}$ Films with less

\footnotetext{
a) Tel.: +46 18471 3101. FAX: +46 500131. Electronic mail: gunnar.niklasson@angstrom.uu.se
}

oxygen would then have more $\mathrm{W}^{4+}$ and less $\mathrm{W}^{6+}$ states as compared to stoichiometric films. During intercalation, $\mathrm{W}^{5+}$ states are created, and transitions between the $\mathrm{W}^{4+}$ and the $\mathrm{W}^{5+}$ states give a major contribution to the optical absorption, which increases with the degree of substoichiometry. Hence, the coloration efficiency would increase with increasing substoichiometry, as observed by Lee $e t$ al. ${ }^{10}$ In addition, optical measurements at a single wavelength have been claimed to support this model. ${ }^{11}$ However, the opposite result, namely, the coloration efficiency is highest for stoichiometric tungsten oxide films, has also been reported. ${ }^{12}$

It has been found that substoichiometric films in the $\mathrm{WO}_{y}$ range $(\sim 2.75<y \leqslant 3.0)$ are very transparent, ${ }^{13}$ and a colored (blue) phase is seen only when the nonstoichiometry is substantial $(y<\sim 2.75){ }^{13,14}$ The colored $\mathrm{WO}_{y}$ films exhibit a similar optical absorption spectrum, as compared to intercalated films, which indicates a similar absorption process. ${ }^{15}$ Colored substoichiometric films can also be bleached and colored electrochemically. It has been suggested that transparent substoichiometric $\mathrm{WO}_{y}$ films contain filled gap states associated with $(\mathrm{W}-\mathrm{W})^{10+}$ defects. ${ }^{16}$

It is clear that the influence of defects, as well as the various charge states of the tungsten ions, on the optical properties of amorphous $\mathrm{WO}_{y}$ thin films is far from understood. In this paper, we report on a thorough optical study of lithium intercalated tungsten oxide $\mathrm{Li}_{x} \mathrm{WO}_{y}$, with $y$ between 2.63 and 2.93. In particular, we vary the $\mathrm{Li} / \mathrm{W}$ ratio $x$ between 0 and 2, which means that we cover the whole range where $\mathrm{W}^{5+}$ and $\mathrm{W}^{4+}$ states should be predominant on purely statistical grounds. We compare the measured optical absorption coefficient with theory and simulations, considering the available transitions between different tungsten charge states. In Sec. II below, we describe the experiments and give some information about sputter deposition, film composition, electrochemical equipment, and optical measurement. In Sec. III, the basic site-saturation theory for the optical absorption and our modification of this theory taking into account the possible electron transitions are described. In Sec. IV, we show 
the results and present the absorption coefficient for different film compositions. The total absorption coefficient is compared with our modified site-saturation theory. Preliminary results from the present work have been published recently. ${ }^{17}$

\section{EXPERIMENTS}

Tungsten oxide films were deposited by reactive dc magnetron sputtering in a Balzers UTT deposition system, onto glass substrates precoated with about $40 \mathrm{~nm}$ thick indium tin oxide (ITO), having a resistivity of $60 \Omega / \square$. The sputter chamber was baked for more than $7 \mathrm{~h}$ at $120{ }^{\circ} \mathrm{C}$ and a pressure of around $10 \mu$ Torr before the films were deposited. A 5 -cm-diameter plate of pure tungsten $(99.99 \%)$ was used as the target. The sputter pressure was fixed at 20 mTorr with a power of $200 \mathrm{~W}$, while the oxygen/argon gas flow ratios were either $0.12,0.16$, or 0.44 . The lowest ratio gave a film that, as deposited, was blue, while the others were transparent.

These films have compositions and densities of $\mathrm{WO}_{2.63}$ and $\sim 7.2 \mathrm{~g} / \mathrm{cm}^{3}, \mathrm{WO}_{2.89}$ and $\sim 5.7 \mathrm{~g} / \mathrm{cm}^{3}$, and $\mathrm{WO}_{2.93}$ and $\sim 5.0 \mathrm{~g} / \mathrm{cm}^{3}$, according to earlier measurements by elastic recoil detection analysis on films deposited with identical sputter conditions. ${ }^{13}$ The film thicknesses were measured by a Tencor Alpha-Step stylus profilometer and were found to be $d=(310 \pm 25) \mathrm{nm}$. The films have been submitted to $\mathrm{x}$-ray diffraction analysis, using a Siemens D5000 diffractometer and employing $\mathrm{Cu} K \alpha$ radiation. None of them showed any peaks that could indicate crystallinity; thus, they are said to be x-ray amorphous.

Intercalation of the samples was done electrochemically in an argon filled glove box with humidity of less than $6 \mathrm{ppm}$ by using the chronopotentiometry procedure, that is, by applying a constant current for a given time. The setup consisted of three electrodes where pure lithium foils were used for the counter and reference electrodes while the sample was the working electrode. These were immersed in an electrolyte of $1 M$ lithium perchlorate $\left(\mathrm{LiClO}_{4}\right)$ dissolved in propylene carbonate. The as-deposited blue films were bleached before the first intercalation. The transparent samples were first intercalated, then taken out of the glove box, and washed with ethanol. After performing optical measurements under ambient atmospheric conditions, they were deintercalated in the glove box, in order to regain their original transparency. The deintercalation was carried out by applying the equilibrium potential of the transparent film and monitoring the resulting current. It was found that the charge extracted by this procedure was $5 \%-15 \%$ less than the inserted charge during intercalation. A substantial part of this decrease occurred during the washing of the samples with ethanol. The $\mathrm{Li} / \mathrm{W}$ ratios $(x)$ of the films were computed from the average of the inserted and extracted charges. Hence, they exhibit relative errors of at most $7.5 \%$.

The samples were then reused in the same way but now intercalated with more lithium. This procedure was continued until the intercalated samples could not be deintercalated to transparency anymore, i.e., when optical irreversibility occurred. After that stage, in our case for $\mathrm{Li} / \mathrm{W}$ ratios of above 0.7 , new samples were used for each intercalation level. The maximum intercalation level that could be used was $2 \mathrm{Li} / \mathrm{W}$ $\left(\sim 150 \mathrm{mC} / \mathrm{cm}^{2}\right)$, in agreement with earlier results. ${ }^{18}$ Evidence has been found for electrocrystallization on the surface above $550 \mathrm{mC} / \mathrm{cm}^{2} \mu \mathrm{m} .{ }^{18}$ A measurement series was performed using new samples for each optical measurement also in the optically reversible range. It was established before that results using this procedure coincide with the data for reused samples. ${ }^{19}$

An ultraviolet/visible/near infrared Perkin-Elmer Lambda 9 spectrophotometer, with an integrating sphere coated with a highly diffusely reflecting barium sulfate $\left(\mathrm{BaSO}_{4}\right)$ paint, was used for optical transmittance and reflectance measurements at wavelengths between 300 and $2500 \mathrm{~nm}(4.1-0.5 \mathrm{eV})$. A plate of barium sulfate was used as reference for the reflectance measurements. The optical spectra of our intercalated tungsten oxide films changed slowly when the samples were exposed to ambient atmosphere. This effect will lead to serious measurement errors for exposure times of $30 \mathrm{~min}$ and longer. Between the intercalation and deintercalation, our samples were exposed for less than $15 \mathrm{~min}$ to the ambient, leading to negligible effects on the optical spectra.

\section{THEORY}

In order to formulate a theory of optical absorption in amorphous tungsten oxide, we must first discuss which electronic states are involved in the optical transitions. For subband-gap absorption the alternatives are defect states in the band gap and states in the conduction band. Although defect states cannot be discounted, we assume that the main contribution to the optical absorption comes from transitions between band states. The number of electrons inserted into the films during intercalation is equal to $x$ and, hence, at most $2 /$ f.u. of $\mathrm{WO}_{y}$. As more and more electrons are inserted, the Fermi level will emerge from the band gap and rise higher and higher into the conduction band. Recent studies of the density of states (DOS) of amorphous tungsten oxide by intercalation spectroscopy ${ }^{20}$ showed that the inserted electrons enter conduction band states. The effective DOS obtained from chronopotentiometry measurements displays an excellent agreement with the computed conduction band DOS for monoclinic tungsten oxide. ${ }^{20}$ The electrical conductivity of amorphous tungsten oxide films follows the variable range hopping behavior, at least up to $x \sim 0.5,{ }^{21}$ indicating that the states in the lower part of the conduction band are localized. The mobility edge appears to be around $1.3 \mathrm{eV}$ from the band edge. ${ }^{22}$

Theories for optical absorption due to transitions between localized states have been proposed based on various theoretical frameworks. In the present context, the most relevant ones appear to be intervalence transfer absorption ${ }^{23}$ and small polaron absorption, ${ }^{24-26}$ which have both been used for tungsten oxide films. ${ }^{7,19,27}$ These theories lead to quite similar expressions and appear to be difficult to distinguish from one another. A common factor is that the optical absorption, described in terms of the real part of the optical conductivity or the absorption coefficient, can be accurately modeled by Gaussian peak profiles. This is easily demon- 
strated by using, for example, the relations of Böttger and Bryksin $^{26}$ for the real and imaginary parts of the optical conductivity.

In the present paper, we do not take the complex details of the electronic band structure into account but instead use a simplified approach. We assume that the optical absorption in amorphous tungsten oxide can be decomposed into three components due to the three possible intervalence transitions between $\mathrm{W}^{6+}, \mathrm{W}^{5+}$, and $\mathrm{W}^{4+}$ sites. The contribution to the optical absorption coefficient from each intervalence transition process is modeled as a Gaussian peak. Our theory is a generalization of the site-saturation model of Denesuk and Uhlmann. $^{28}$

The original site-saturation model assumes that the optical absorption coefficient is proportional to the number of available electronic transitions from a $\mathrm{W}^{5+}$ ion to a $\mathrm{W}^{6+}$ one. Let $p$ be the probability that an inserted electron becomes situated at a certain $\mathrm{W}^{6+}$ site. The number of transitions is found by multiplying the probabilities of a site being $\mathrm{W}^{6+}$ $(1-p)$ and $\mathrm{W}^{5+}(p)$ and is given by. ${ }^{28}$

$$
\mathrm{W}^{5+} \leftrightarrow \mathrm{W}^{6+}, \quad P=p-p^{2} .
$$

This equation predicts that the maximum of the total absorption should be at $p=0.5$. In the case of stoichiometric tungsten oxide, $p=x$. The model can easily be generalized to $\mathrm{Li}_{x} \mathrm{WO}_{3-z}$ films by adding an assumption about the defects that are present. We find now that

$$
p=x /(1-A z),
$$

where $A=2$ if the oxygen deficiency is due to optically inactive $(\mathrm{W}-\mathrm{W})^{10+}$ defects and $A=1$ if it is due to $\mathrm{W}^{4+}$ ions. Equation (2) holds in these two cases with the restriction that $z<0.5$ and $z<1$, respectively. It is realized that the absorption maximum should shift toward lower values of $x$ as the oxygen deficiency $(z)$ increases. In the $A=1$ case, transitions between $\mathrm{W}^{4+}$ and $\mathrm{W}^{5+}$ with $P=[z /(1-z)] x$ will obviously also be present.

It is possible to generalize the site-saturation model to also include $\mathrm{W}^{4+}$ states besides the $\mathrm{W}^{6+}$ and the $\mathrm{W}^{5+}$ states. For this reason we assume that each site can be "empty" or "filled" not only with one electron but also with two. Before intercalation starts all sites are taken to be empty. Starting with the insertion of electrons, most of the occupied sites will be singly occupied in the beginning. Electron transitions between empty and singly occupied states are then the most common. As more singly occupied sites are filled there will be an increased probability that also doubly occupied sites will be formed. The probabilities of a site being $\mathrm{W}^{4+}, \mathrm{W}^{5+}$, and $\mathrm{W}^{6+}$ are then $p^{2}, 2 p(1-p)$, and $(1-p)^{2}$, respectively.

Analytical expressions for the possible electronic transitions are given by

$$
\begin{aligned}
& \mathrm{W}^{6+} \leftrightarrow \mathrm{W}^{5+}, \quad P=2 p(1-p)^{3}, \\
& \mathrm{~W}^{5+} \leftrightarrow \mathrm{W}^{4+}, \quad P=2 p^{3}(1-p), \\
& \mathrm{W}^{6+} \leftrightarrow \mathrm{W}^{4+}, \quad P=p^{2}(1-p)^{2} .
\end{aligned}
$$

These equations exhibit maxima at $p=0.25,0.75$, and 0.5 , respectively. For the case of stoichiometric tungsten oxide

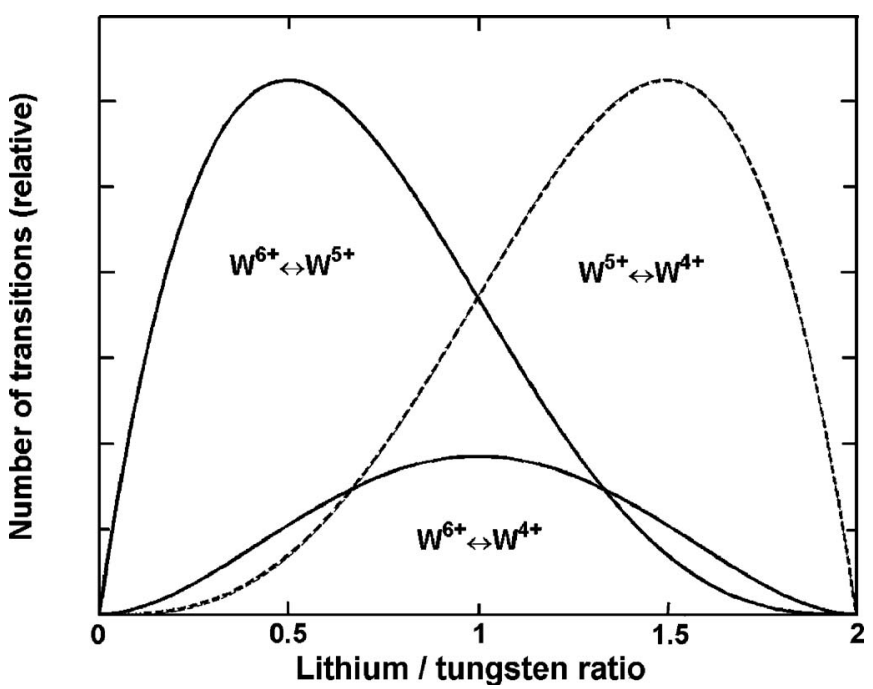

FIG. 1. Relative number of transitions for the transitions $\mathrm{W}^{6+} \leftrightarrow \mathrm{W}^{5+} \mathrm{W}^{5+} \leftrightarrow \mathrm{W}^{4+}$, and $\mathrm{W}^{6+} \leftrightarrow \mathrm{W}^{4+}$ as a function of $\mathrm{Li} / \mathrm{W}$ ratio, given by computer simulations and the analytic expressions in Eqs. (3)-(5).

films, $p=x / 2$, while for substoichiometric films, one should use $p=x /[2(1-A z)]$. Also in this case, the maxima will move to lower values of $x$ as $z$ increases.

These analytical expressions have been verified by computer simulations, for the case of $\mathrm{Li}_{x} \mathrm{WO}_{3}$ films. The simulation represents the sites in the material with a $100 \times 100$ $\times 100$ matrix. Each element in the matrix can have the values of 0,1 , or 2 electrons, corresponding to charge states of $6+, 5+$, and 4+, respectively. The matrix is empty at the beginning of the simulation, corresponding to a $\mathrm{Li} / \mathrm{W}$ ratio of 0 . A random site then has its value increased by 1 . This changes the state of the site, affecting the total number of possible different transitions. The number of possible transitions is recorded for each inserted electron. The procedure is repeated until all sites have two electrons.

A comparison between Eqs. (3)-(5) and the simulations is depicted in Fig. 1. The number of transitions between $\mathrm{W}^{6+}$ and $\mathrm{W}^{5+}$ sites is peaked at $x=0.5$ as expected, but the curve is broadened toward 2 (the maximum $x$ value). The $\mathrm{W}^{5+}-\mathrm{W}^{4+}$ transition peak is the mirror image, with respect to $x=1$ of the former one, while the $\mathrm{W}^{6+}-\mathrm{W}^{4+}$ transition peak is completely symmetric. It should be noted that the analytical solution and the computer simulations are in excellent agreement with each other, so that differences are not visible in Fig. 1. It should also be realized that the absorption strength per transition could well be different in the three cases.

The site-saturation model does not give any information of the peak energies of the three transitions in Eqs. (3)-(5), for which we expect Gaussian peak profiles as discussed above. We will infer the energies from comparison of the model with experimental data in Sec. IV below.

\section{RESULTS}

Figure 2 shows measurements of the reflectance and the transmittance on as-deposited and $\mathrm{Li}$ intercalated $\mathrm{Li}_{x} \mathrm{WO}_{2.89}$ films, for values of $x$ up to 0.45 . The as-deposited film shows a very good transparency. It is seen that the transmittance 

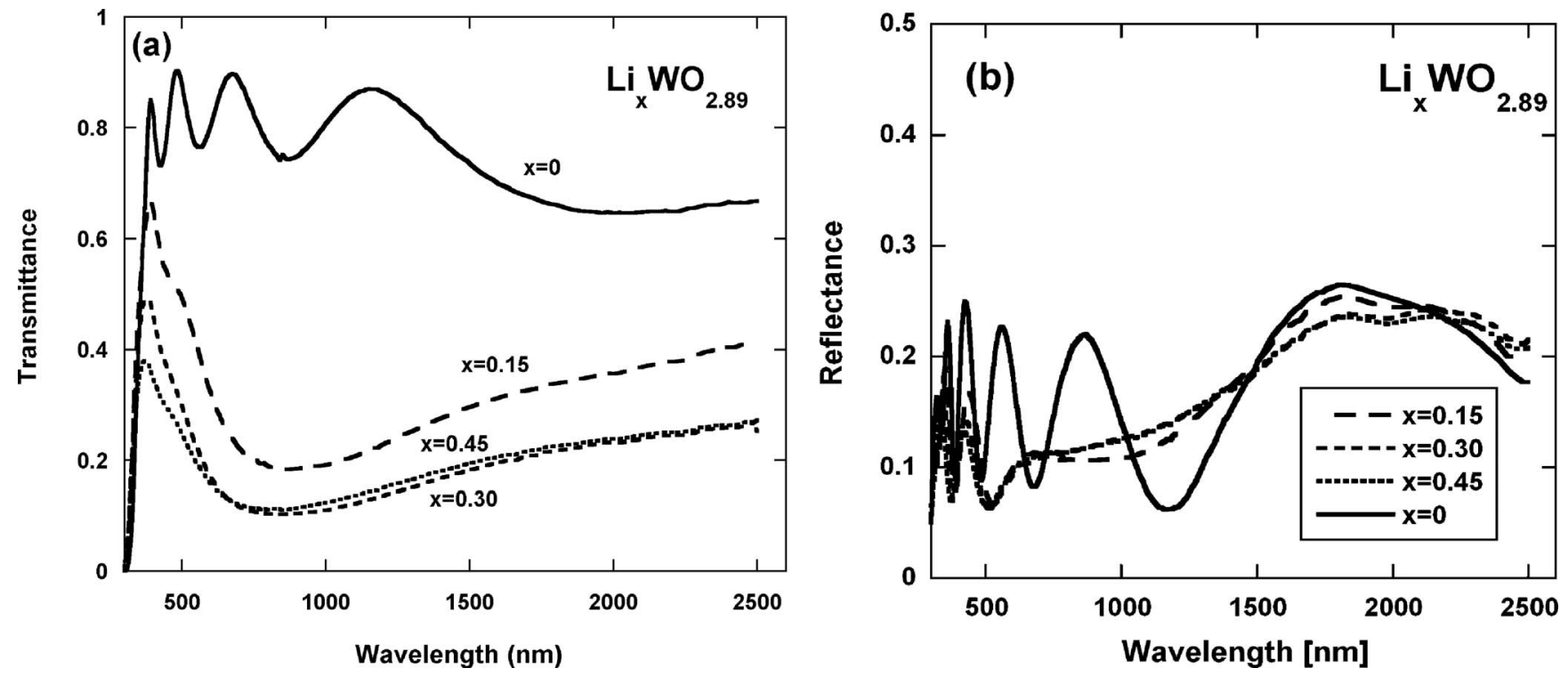

FIG. 2. Transmittance (a) and reflectance (b) as a function of wavelength for $\mathrm{Li}_{x} \mathrm{WO}_{2.89}$ as-deposited $(x=0)$ and intercalated films. The values of $x$ are given in the figure.

decreases as intercalation proceeds and exhibits a broad minimum for $x$ between 0.3 and 0.5 . On the other hand, the magnitude of the reflectance does not change much, but the interference is suppressed as $x$ increases. In this range of $\mathrm{Li}$ content, the films exhibit a striking blue color. Figure 3 shows transmittance and reflectance spectra as more $\mathrm{Li}$ ions are intercalated into the films. For $x>0.6$ the transmittance starts to increase and films with $x$ approaching 2 are almost completely transparent for wavelengths above $1 \mu \mathrm{m}$. The lowering of the transmittance toward shorter wavelengths, as seen in the figure, gives the films a light-brown color. The results indicate that the absorption decreases after saturation at $x \sim 0.5$, during the intercalation. This will be discussed in more detail below.

Reflectance $(R)$ and transmittance $(T)$ were measured on three sets of lithium intercalated films with different compositions $\left(\mathrm{WO}_{2.63}, \mathrm{WO}_{2.89}\right.$, and $\left.\mathrm{WO}_{2.93}\right)$. The transmittances of

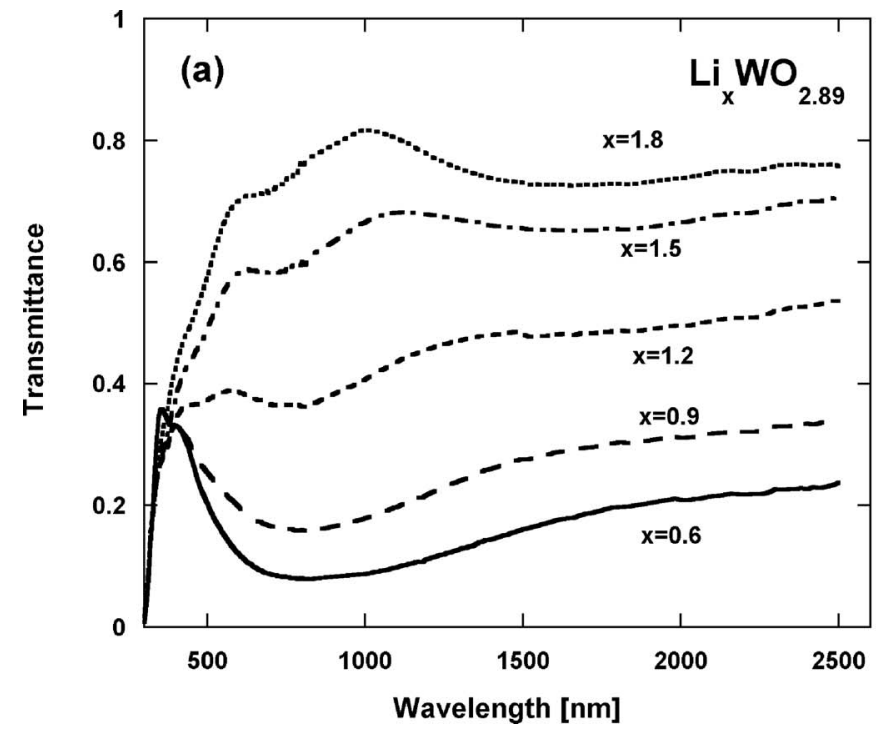

all three sets of films showed features qualitatively similar to those in Figs. 2 and 3. The absorption coefficient $(\alpha)$, as a function of energy $(E)$ in the range of 0.5 to $4 \mathrm{eV}$, was calculated according to the expression: ${ }^{29}$

$$
\alpha(E)=\frac{1}{d} \ln \frac{1-R(E)}{T(E)},
$$

where $d$ is the film thickness. This equation has been shown to give values in good agreement with direct $R-T$ inversion. ${ }^{30}$ It is by no means certain that the $\mathrm{Li}$ concentration and, hence, the optical absorption are uniform throughout the film. At least for low values of $x$, the front and pore surfaces in the film might be more likely to exhibit a higher Li concentration.

In Fig. 4(a), we display the absorption coefficients $(\alpha)$ of a lithium intercalated $\mathrm{WO}_{2.89}$ film at various intercalation

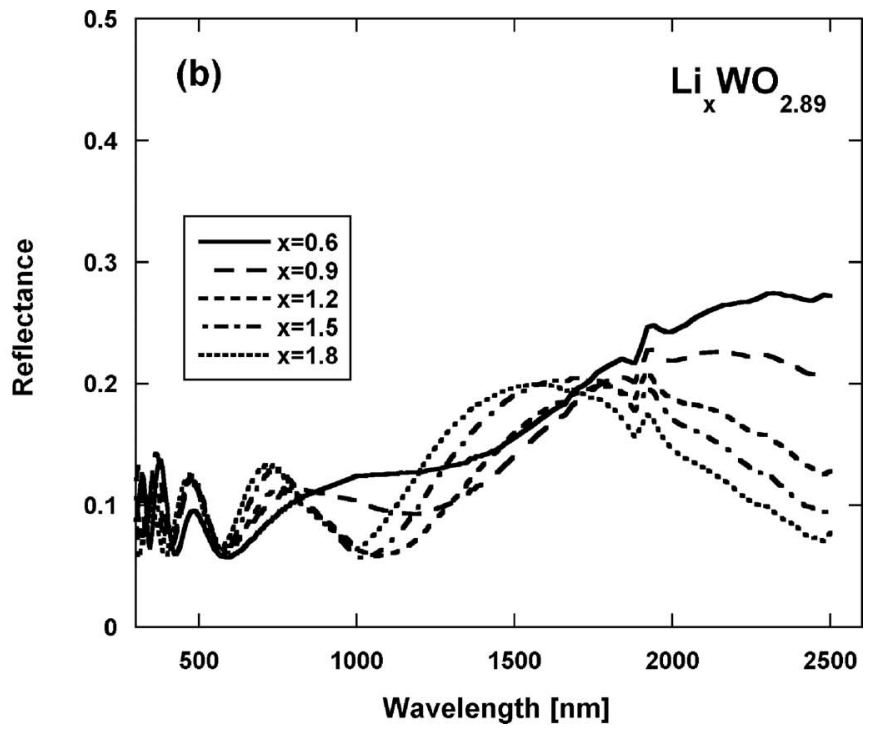

FIG. 3. Transmittance (a) and reflectance (b) as a function of wavelength for $\mathrm{Li}_{x} \mathrm{WO}_{2.89}$ heavily intercalated films. The values of $x$ are given in the figure. 

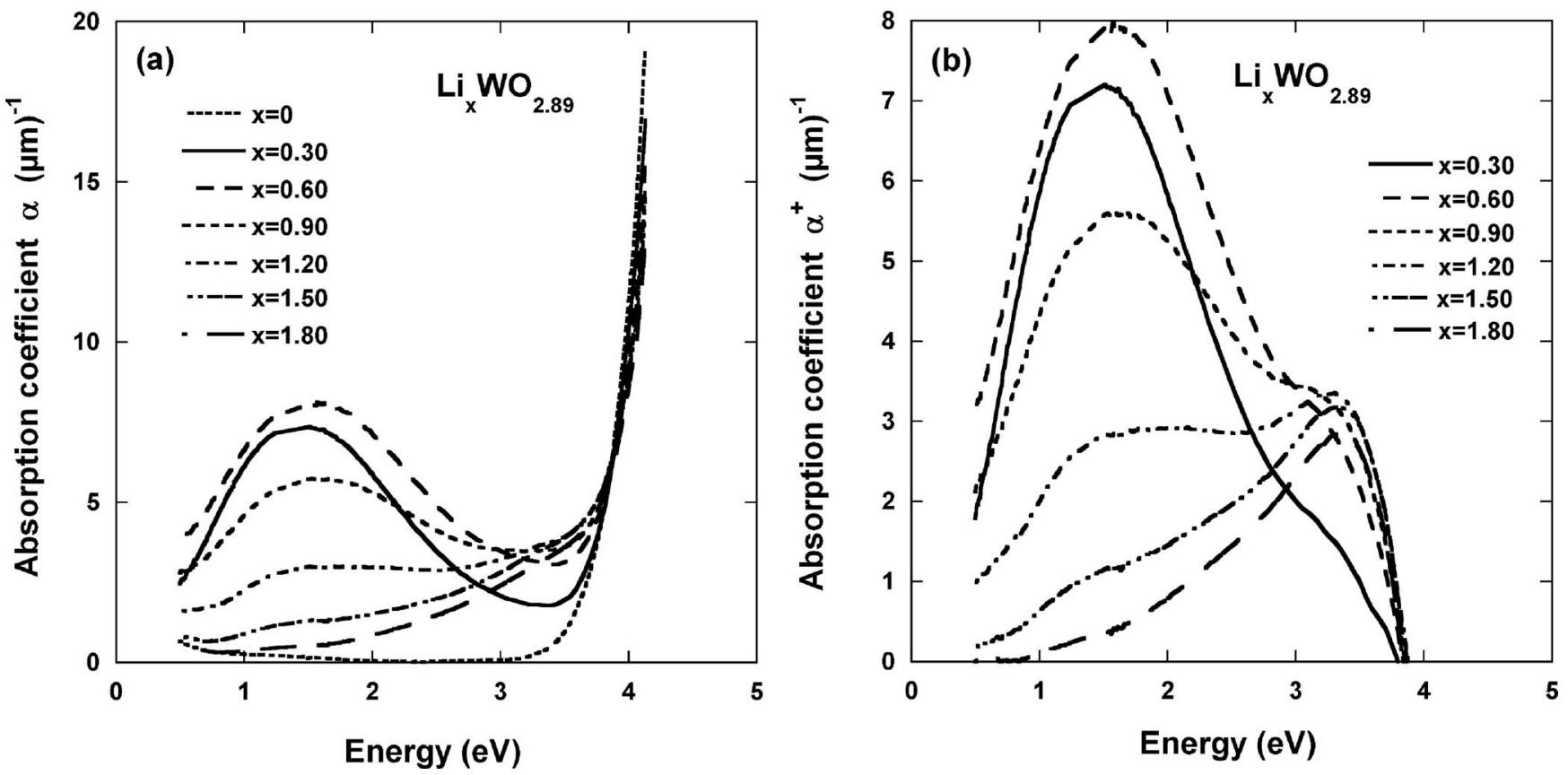

FIG. 4. The absorption coefficients, $\alpha(E)$, for substoichiometric amorphous $\mathrm{Li}_{x} \mathrm{WO}_{2.89}$ films for different intercalation levels as a function of energy (a) and absorption coefficients, $\alpha^{+}(E)$, obtained by subtraction of the absorption coefficient of the nonintercalated film $[x=0$ in (a) (b). The values of $x$ are given in the figure.

levels as a function of energy. The steeply rising $\alpha$ above $3.5 \mathrm{eV}$ is due to interband transitions over the fundamental band gap of tungsten oxide. The absorption in the asdeposited film $(x=0)$ is close to zero and the slight increase toward low energies is due to absorption in the ITO coating on the substrate. In this paper, we concentrate on the optical subband-gap absorption in tungsten oxide. To analyze this contribution, we subtract $\alpha$ for the nonintercalated film from the $\alpha$ 's of the intercalated ones. The subtracted $\alpha$ is shown in Fig. 4(b) and is denoted by $\alpha^{+}$. It can be observed that the absorption coefficient displays a maximum at $\sim 1.4 \mathrm{eV}$, which first increases as the intercalation level increases. Then, this peak decreases after reaching a saturation value. It decreases at the same time as another peak is revealed at a higher energy. This continues until the new peak at $\sim 3.3 \mathrm{eV}$ has taken over most of the absorption. The new peak is responsible for the brown color of the films, observed when the intercalation level is above $0.7 \mathrm{Li} / \mathrm{W}$.

The total optical absorption in the films will now be considered. The total absorption in the measurement range was obtained by integration of the absorption coefficient. The integration was performed in two ways. The first was a straightforward integration, while the second was carried out by dividing the absorption coefficient with the energy and then integrating this expression over the energy range. The two integrations are mathematically expressed as follows:

$$
\begin{aligned}
& \alpha_{\mathrm{TOT}}^{*}(E)=\int \alpha(E) d E, \\
& \alpha_{\mathrm{TOT}}(E)=\int \frac{\alpha(E)}{E} d E .
\end{aligned}
$$

The first equation gives the total absorbed energy per unit thickness, while the second gives the total absorption coeffi- cient. Figure 5 gives these quantities as a function of the $x$ value between 0 and 2 for a $\mathrm{Li}_{x} \mathrm{WO}_{2.89}$ film. There are no notable differences between the results from the two methods except that the curves are shifted vertically.

Figure 6 shows the total absorption coefficient derived from Eq. (8) for samples with different compositions, a $\mathrm{WO}_{2.93}$ film as well as the more substoichiometric $\mathrm{WO}_{2.89}$ and $\mathrm{WO}_{2.63}$ films. In this figure, a vertical line is drawn at $x \sim 0.7 \mathrm{Li} / \mathrm{W}$ to show where the films could not be made transparent by electrochemical deintercalation anymore. The figure shows that the total absorption of the three films can be approximated by a polynomial equation of second degree

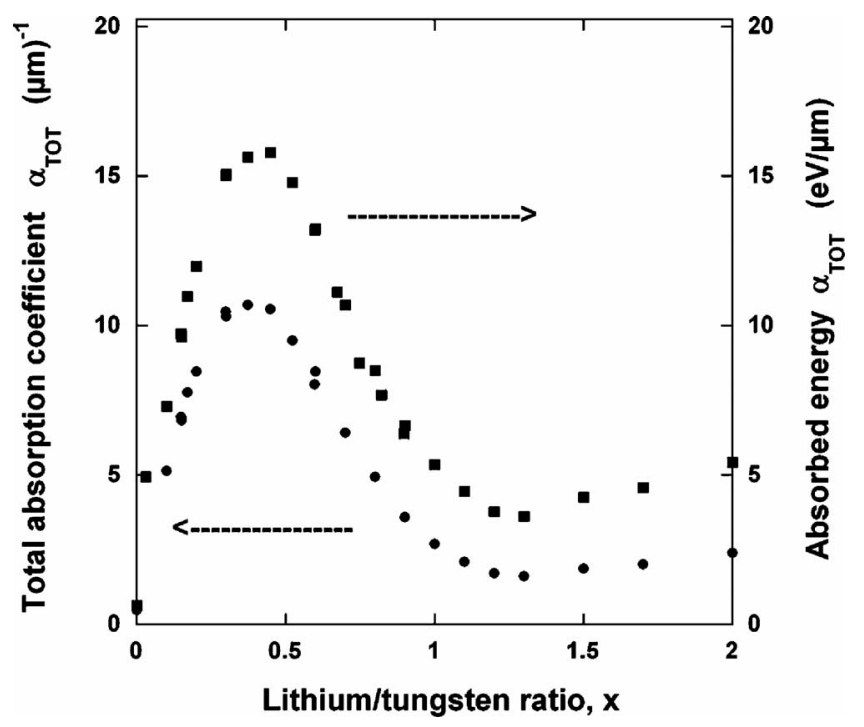

FIG. 5. The total integrated absorption expressed in two ways: Total ab-

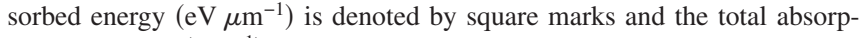
tion coefficient $\left(\mu \mathrm{m}^{-1}\right)$ is denoted by circles. Data are given for a $\mathrm{Li}_{x} \mathrm{WO}_{2.89}$ film as a function of $x$. 


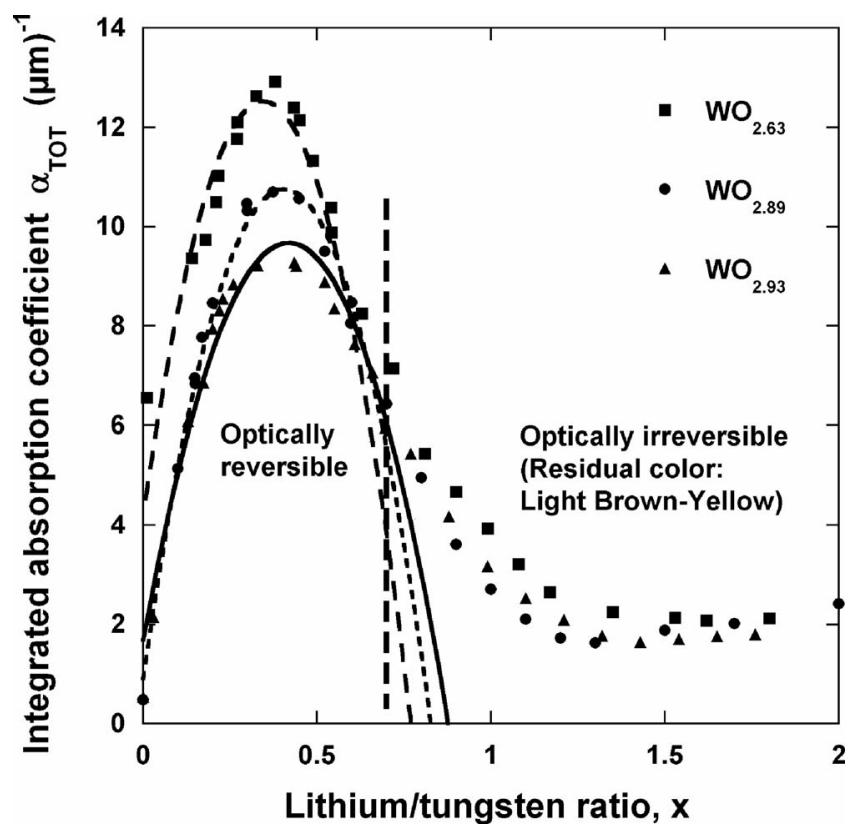

FIG. 6. The total absorption coefficient as a function of lithium intercalation level $(x)$ for $\mathrm{Li}_{x} \mathrm{WO}_{2.63}, \mathrm{Li}_{x} \mathrm{WO}_{2.89}$, and $\mathrm{Li}_{x} \mathrm{WO}_{2.93}$ films. The dashed vertical line divides optically reversible inter/deintercalation from nonreversible ones. The irreversible films showed a residual color of light yellow brown after bleaching.

according to the site-saturation theory [Eq. (1)]. The experimental points are in good agreement with the fit up to the vertical reversible/irreversible demarcation line. There is a tendency that the maximum of the total absorption shifts to lower intercalation levels as the $\mathrm{O} / \mathrm{W}$ ratio decreases, as expected from Eqs. (1) and (2). It can be seen that the total absorption coefficient is highest for the $\mathrm{WO}_{2.63}$ film and decreases as the $\mathrm{O} / \mathrm{W}$ ratio increases. The values at the peak maximum scale approximately with the measured densities of the films (compare with Sec. II). Hence, it is not necessary to invoke $\mathrm{W}^{4+}$ sites $^{8-10}$ to explain this trend.

The features of Fig. 4(b) make it natural to try to resolve the two peaks in the spectrum. However, by using two Gaussian peaks, completely satisfactory fits were not obtained. We have found it necessary to use three peaks, in order to obtain a good fit to absorption spectra for all $\mathrm{Li} / \mathrm{W}$ ratios $(x)$. The model with two peaks was less satisfactory, although it yielded equally good fits at low and high values of $x$. Two of the peaks were fixed at their experimentally found positions $1.4 \mathrm{eV}$ (peak 1) and $3.37 \mathrm{eV}$ (peak 3), while the third peak position (peak 2) was obtained from the fitting and was found to vary between 2.45 and $2.70 \mathrm{eV}$. The dependence on $x$ of the strengths of the Gaussian peaks was computed from the best fits to the spectra.

Figure 7 shows the total absorption coefficient, decomposed into the three peaks, as a function of the $x$ value for the tungsten oxide films $\mathrm{WO}_{2.93}(\mathrm{a}), \mathrm{WO}_{2.89}(\mathrm{~b})$, and $\mathrm{WO}_{2.63}$ (c). It is seen that peak 1 is the strongest one and it has its maximum at an $x$ value of around 0.5 for all the films. The peak increases as the oxygen deficiency increases. By com-
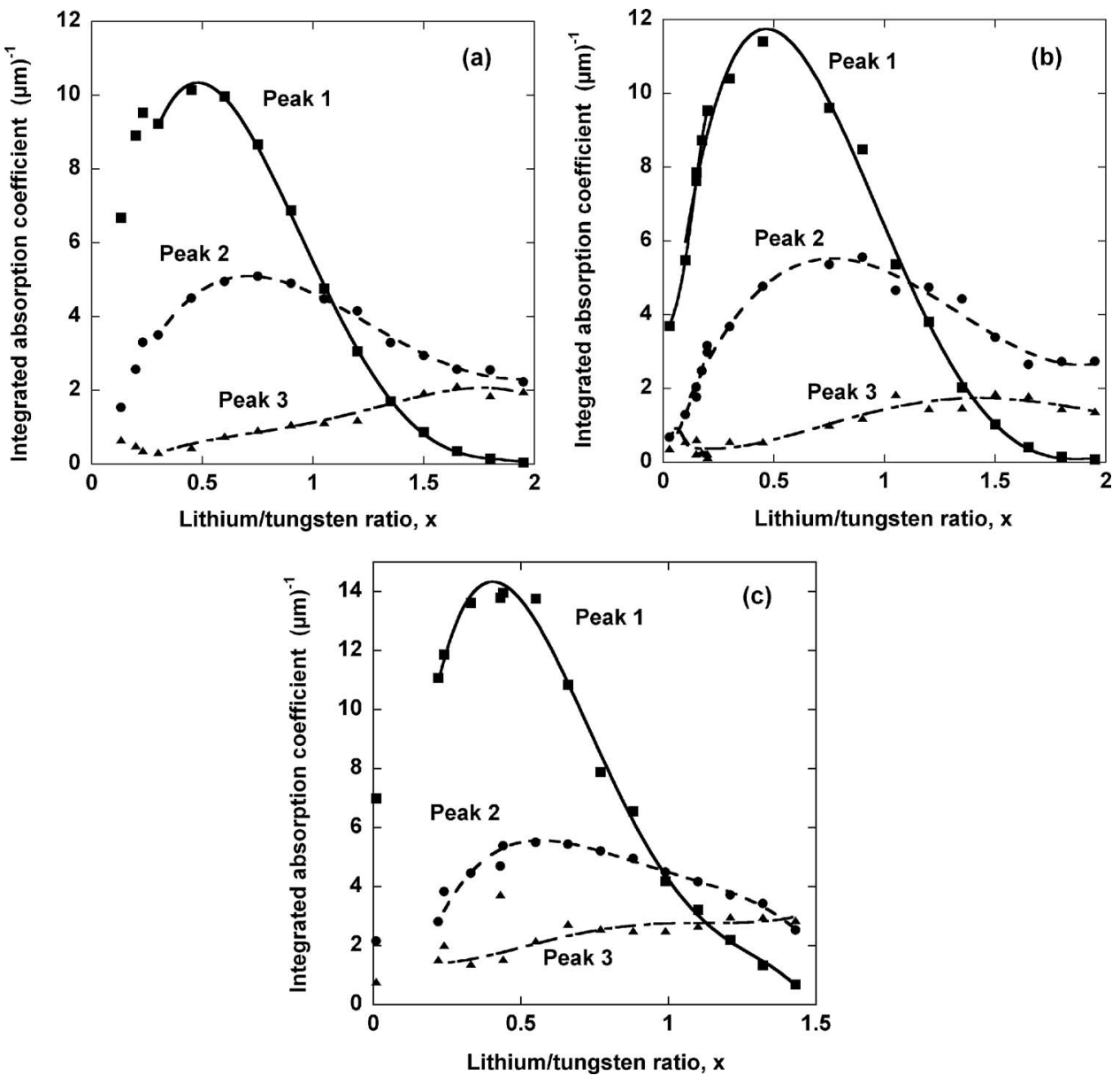

FIG. 7. Integrated total absorption coefficients as a function of $x$ for $\mathrm{Li}$ intercalated amorphous tungsten oxide films. The strength of three superpositioned Gaussian peaks, positioned at energies of $1.4 \mathrm{eV}$ (peak 1), $3.37 \mathrm{eV}$ (peak 3), and 2.45-2.70 eV (peak 2), were found by a fit to the absorption coefficient shown in Fig. 4(b). The data pertain to $\mathrm{Li}_{x} \mathrm{WO}_{2.93} \quad$ (a), $\mathrm{Li}_{x} \mathrm{WO}_{2.89}$ (b), and $\mathrm{Li}_{\mathrm{x}} \mathrm{WO}_{2.63}$ (c) films. 
parisons with the computations in Fig. 1, we designate this peak to $\mathrm{W}^{5+} \leftrightarrow \mathrm{W}^{6+}$ transitions.

The parameters for the other two peaks are more uncertain, since almost good fits could be obtained with a range of values. However, the tendency is clear and shows a marked similarity with the calculations in Fig. 1. Peak 3, which was fixed at $3.37 \mathrm{eV}$, increases continuously as the intercalation level increases up to $x=1.5$ and it is higher for the $\mathrm{WO}_{2.63}$ film than for the others. Peak 2, the position of which was allowed to vary during the fitting, is pronounced and it seems to be at a maximum between $x$ values of 0.5 and 1. Clearly, we should designate peak 2 to $\mathrm{W}^{4+} \leftrightarrow \mathrm{W}^{6+}$ transitions and peak 3 to $\mathrm{W}^{4+} \leftrightarrow \mathrm{W}^{5+}$ transitions. Hence, there is a significant behavior of the three peaks, that is, in qualitative agreement with a modified site-saturation model.

It appears difficult to relate the energies at which the peaks are centered to features of the electron band structure. Since the Fermi level increases with $\mathrm{Li} / \mathrm{W}$ ratio $x$, transition energies might be expected to vary with $x$, if features in the band structure are important. However, the energies of the three peaks do not seem to depend on the degree of intercalation, within experimental accuracy. This indicates that an improved understanding of the detailed dynamics of the different transitions appears to be necessary for establishing a relation between the optical properties and the electronic structure of amorphous tungsten oxide films.

\section{CONCLUSIONS}

We have studied the optical absorption in intercalated tungsten oxide films in a very large range of Li concentration. We found that the optical absorption profile can be decomposed into three peaks. One of them is related to the normal optically reversible electrochromic absorption and appears at intercalation levels below about $0.7 \mathrm{Li} / \mathrm{W}$. Another one, which exhibits an optically irreversible absorption, appears at a $\mathrm{Li} / \mathrm{W}$ ratio of approximately 0.7 . The third one can only be resolved by computer fitting. The dependence of the integrated strength of the peaks on intercalation level shows striking similarities with a generalized site-saturation model. We identify the features in the optical spectra as being due to $\mathrm{W}^{5+} \leftrightarrow \mathrm{W}^{6+}, \mathrm{W}^{4+} \leftrightarrow \mathrm{W}^{6+}$, and $\mathrm{W}^{4+} \leftrightarrow \mathrm{W}^{5+}$ transitions.

\section{ACKNOWLEDGMENTS}

This work was supported by a grant from the Swedish Science Council (VR). We are grateful for valuable discussions with C. G. Granqvist. We also want to thank J. Backholm for help with computer software.

${ }^{1}$ C. M. Lampert, Sol. Energy Mater. Sol. Cells 76, 489 (2003).

${ }^{2}$ G. A. Niklasson and C. G. Granqvist, J. Mater. Chem. 17, 127 (2007).

${ }^{3}$ E. Avendano, A. Azens, G. A. Niklasson, and C. G. Granqvist, Mater. Sci. Eng., B 138, 112 (2007).

${ }^{4}$ C. G. Granqvist, Handbook Of Inorganic Electrochromic Materials (Elsevier, Amsterdam, 1995).

${ }^{5}$ C. G. Granqvist, Sol. Energy Mater. Sol. Cells 60, 201 (2000).

${ }^{6}$ S. K. Deb, Philos. Mag. 27, 801 (1973).

${ }^{7}$ B. W. Faughnan, R. S. Crandall, and P. M. Heyman, RCA Rev. 36, 177 (1975).

${ }^{8}$ J.-G. Zhang, D. K. Benson, C. E. Tracy, S. K. Deb, A. W. Czanderna, and C. Bechinger, J. Electrochem. Soc. 144, 2022 (1997).

${ }^{9}$ C. Bechinger, M. S. Burdis, and J.-G. Zhang, Solid State Commun. 101, 753 (1997).

${ }^{10}$ S.-H. Lee, H. M. Cheong, C. E. Tracy, A. Mascharenhas, A. W. Czanderna, and S. K. Deb, Appl. Phys. Lett. 75, 1541 (1999).

${ }^{11}$ M. Stolze, D. Gogova, and L.-K. Thomas, Thin Solid Films 476, 185 (2005).

${ }^{12}$ A. Subramanyam, A. Karuppasamy, and C. Suresh Kumar, Electrochem. Solid-State Lett. 9, H111 (2006).

${ }^{13}$ L. Berggren and G. A. Niklasson, Sol. Energy Mater. Sol. Cells 85, 573 (2005).

${ }^{14}$ P. Gerard, A. Deneuville, G. Hollinger, and T. M. Duc, J. Appl. Phys. 48, 4252 (1977).

${ }^{15}$ L. Berggren and G. A. Niklasson, Solid State Ionics 165, 51 (2003).

${ }^{16}$ G. A. de Wijs and R. A. de Groot, Phys. Rev. B 60, 16463 (1999).

${ }^{17}$ L. Berggren and G. A. Niklasson, Appl. Phys. Lett. 88, 081906 (2006).

${ }^{18}$ J. Isidorsson, T. Lindström, C. G. Granqvist, and M. Herranen, J. Electrochem. Soc. 147, 2784 (2000).

${ }^{19}$ L. Berggren and G. A. Niklasson, J. Appl. Phys. 90, 1860 (2001).

${ }^{20}$ M. Strömme, R. Ahuja, and G. A. Niklasson, Phys. Rev. Lett. 93, 206403 (2004).

${ }^{21}$ L. Berggren, J. Ederth, and G. A. Niklasson, Sol. Energy Mater. Sol. Cells 84, 329 (2004).

${ }^{22}$ G. A. Niklasson, A. K. Norling, and L. Berggren, "Charge transport between localized states in lithium-intercalated amorphous tungsten oxide," J. Non-Cryst. Solids (in press).

${ }^{23}$ N. S. Hush, Prog. Inorg. Chem. 8, 391 (1967).

${ }^{24}$ I. G. Austin and N. F. Mott, Adv. Phys. 18, 41 (1969).

${ }^{25}$ O. F. Schirmer, P. Koidl, and H. G. Reik, Phys. Status Solidi B 62, 385 (1974).

${ }^{26} \mathrm{H}$. Böttger and V. V. Bryksin, Hopping Conduction in Solids (VCH, Weinheim, 1985), Chap. 2.

${ }^{27}$ O. F. Schirmer, V. Wittwer, G. Baur, and G. Brandt, J. Electrochem. Soc. 124, 749 (1977).

${ }^{28}$ M. Denesuk and D. R. Uhlmann, J. Electrochem. Soc. 143, L186 (1996).

${ }^{29}$ W. Q. Hong, J. Phys. D 22, 1384 (1989).

${ }^{30}$ J. Rodriguez, M. Gomez, J. Ederth, G. A. Niklasson, and C. G. Granqvist, Thin Solid Films 365, 119 (2000). 